\title{
$\underline{\underline{n}}$ Biblos-e Archivo
}

Repositorio Institucional de la Universidad Autónoma de Madrid

https://repositorio.uam.es

Esta es la versión de autor del artículo publicado en:

This is an author produced version of a paper published in:

Chromsoma 129.1 (2017): 179-194

DOI:http://dx.doi.org/10.1007/s00412-016-0577-6

Copyright: @ Springer-Verlag Berlin Heidelberg 2016

Access to the published version may require subscription

El acceso a la versión del editor puede requerir la suscripción del recurso 


\section{Transcription reactivation during the first meiotic prophase in bugs is not dependent on synapsis}

Alberto Viera 4

María Teresa Parra ${ }^{1}$

Julio S. Rufas 1,2*

Email julio.s.rufas@uam.es

Jesús Page 1,2,

Email jesus.page@uam.es

4 Departamento de Biología, Facultad de Ciencias, Universidad Autónoma de Madrid, Madrid, 28049 Spain AQ1

z Departamento de Biología, Edificio de Ciencias Biológicas, C/Darwin 2, Universidad Autónoma de Madrid, Madrid, 28049 Spain

\section{Abstract}

During meiosis, transcription is precisely regulated in relation to the process of chromosome synapsis. In mammals, transcription is very low until the completion of synapsis in early pachytene, and then reactivates during mid pachytene, up to the end of diplotene. Moreover, chromosomes or chromosomal regions that do not achieve synapsis undergo a specific process of inactivation called meiotic silencing of unpaired chromatin (MSUC). Sex chromosomes, 
which are mostly unsynapsed, present a special case of inactivation named meiotic sex chromosome inactivation (MSCI). Although processes that are similar to MSUC/MSCI have been described in other species like Sordaria and Caenorhabditis elegans, very few studies have been developed in insects. We present a study on the relationships between synapsis and transcription in two hemipteran species (Graphosoma italicum and Carpocoris fuscispinus) that possess holocentric chromosomes but develop different synaptic patterns. We have found that transcription, revealed by the presence of RNA polymerase II, is very low at the beginning of meiosis, but robustly increases during zygotene, long before the completion of synapsis, excepting in the sex chromosomes. In fact, we show that histone H3 methylation at lysine 9 (H3K9me3) may be present in the sex chromosomes at leptotene, thus acting as a likely epigenetic mark for this inactive state. Our results suggest that the meiotic transcription in these two species is differently regulated from that of mammals and, therefore, offer new opportunities to understand the relationship between synapsis and transcription and the mechanisms that govern MSUC/MSCI processes.

\section{Keywords}

Meiosis

Synapsis

Transcription

MSUC

MSCI

\section{Electronic supplementary material}

The online version of this article (doi: 10.1007/s00412-016-0577-6 ) contains supplementary material, which is available to authorized users.

\section{Introduction}

Meiosis is a complex cell division that involves homologous pairing, synapsis, recombination and segregation of 
chromosomes, in order to ensure the proper formation of haploid cells. The chromatin also shows a specific behaviour in relation to condensation, epigenetic regulation and transcription activity (Kota and Feil, 2010 ; Matsui and Hayashi, 2007 ; Page et al., 2012 ; van der Heijden et al., 2007 ). During meiosis, gene expression is known to be tightly regulated in a stage-dependent manner. In mammals, transcriptional activity is particularly reduced during the initial stages of prophase I, reactivates from mid pachytene up to diplotene (Kierszenbaum and Tres, 1974 ; Monesi, 1964 ; Page et al., 2012 ) and is again repressed in metaphase-I until the end of the second meiotic division. In males, transcription is also reactivated during early spermatid differentiation and decreases at later stages of spermiogenesis.

Low transcriptional rate during leptotene and zygotene is concomitant with the progression of synapsis between homologous chromosomes. Thus, it has been inferred that transcription might depend on synapsis completion. In fact, unsynapsed regions during pachytene trigger a process of transcriptional silencing (MSUC, meiotic silencing of unsynapsed chromosomes) (Baarends et al., 2005 ; Manterola et al., 2009 ; Turner et al., 2005 ). This is the case of mammalian sex chromosomes that, in males, present large unsynapsed regions and show MSCI (meiotic sex chromosome inactivation). Both MSUC and MSCI are accompanied by a complex series of epigenetic changes that involve histone modifications, specific histone variants and association of specific proteins and RNAs (Costa et al., 2006; Handel, 2004 ; Hoyer-Fender, 2003 ; Manterola et al., 2009 ; Page et al., 2012 ; Turner, 2007 ; van der Heijden et al., 2007 ). On these grounds, it has been postulated that synapsis is necessary for the transcriptional activity of chromosomes during prophase I (Schimenti, 2005 ). However, the reactivation of transcription does not immediately follow the completion of synapsis between homologues, as indicated by the low transcription rate during early pachytene (Page et al., 2012 ). Moreover, transcriptional repression has also been related to DNA repair (Inagaki et al., 2010 ; Page et al., 2012 ). Interestingly, the phosphorylated form of histone $\mathrm{H} 2 \mathrm{AX}$ at serine $139(\gamma \mathrm{H} 2 \mathrm{AX})$ has been mainly related to the signalling of DNA double-strand breaks (DSBs) in both somatic and meiotic cells (Fernandez-Capetillo et al., 2004 ; Mahadevaiah et al., 2001 ). Thus, the involvement of this histone variant in meiotic silencing could be a consequence of the recruitment of chromatin remodelling factors. Instead, it has been proposed that some other chromatin modifications could act in a more general transcriptional repression program at the beginning of meiosis (Page et al., 2012). Two candidates for promoting this process are the trimethylation of histone $\mathrm{H} 3$ at lysine 9 (H3K9me3) and the 
monomethyation at lysine $4(\mathrm{H} 3 \mathrm{~K} 4 \mathrm{me})$. In mouse, these epigenetic marks appear at the beginning of leptotene and disappear upon transcriptional reactivation during mid pachytene in both synapsed and unsynapsed chromosomes (Page et al., 2012 ; van der Heijden et al., 2007 ).

It is not completely elucidated whether the patterns of epigenetic modifications and transcriptional regulation described in the mouse are applicable to other species. MSUC and MSCI have also been described in other organisms such as Sordaria, Caenorhabditis elegans, grasshoppers and chicken (Kelly and Aramayo, 2007 ; Maine, 2010 ; Schoenmakers et al., 2009 ; Viera et al., 2004b ). In Sordaria, the silencing relies on the activity of small non-coding RNAs (Shiu et al., 2001 ). In C. elegans, the most prominent MSUC mark is H3 dimethyation at lysine 9 (H3K9me2) regulated by siRNA mechanisms (Kelly and Aramayo, 2007 ; Kelly et al., 2002 ; Maine, 2010 ). In grasshoppers, transcription is abundant during the early stages of meiosis and then decreases during pachytene and diplotene (Das et al., 1965 ; Henderson, 1963 ), whereas in Drosophila, transcription is very active throughout the first meiotic prophase in males (Hennig and Weyrich, 2013 ; Kremer et al., 1986 ). Furthermore, chromatin modifications involved in transcription regulation in these insects seem to depart from the histone codes described in mammals (Cabrero et al., 2007 ; Hennig and Weyrich, 2013 ).

In this paper, we have investigated the pattern of transcription activity during meiosis detecting the presence of RNA polymerase II and H3K9me3 in two true bugs, Graphosoma italicum and Carpocoris fuscispinus (Heteroptera: Pentatomidae), that possess the same karyotype and sex chromosomal determinism but differ in the synapsis pattern. In G. italicum, the cohesin axes form in leptotene, appearing entangled over the nucleus, and by zygotene, the axes of homologous chromosomes start to pair. This pairing is rapidly extended along homologues. By contrast, in $C$. fuscispinus, the cohesin axes of homologous chromosomes are formed and appear entangled by leptotene and then gradually approximate during zygotene. However, pairing is not a rapid process. Instead, axes run in parallel along the entire length of homologues with only some discrete points of contact, giving rise to the appearance of bubbles along cohesin axes of homologues. As a consequence, the completion of synapsis in this species appears delayed.

The analysis of both hemipteran species offered a gorgeous opportunity to test in these insects the proposal that transcription reactivation in meiosis might be dependent on synapsis completion. Our results show that transcription in 
these two true bugs is activated during early stages of meiosis, well before synapsis completion. Only the sex chromosomes show patent transcription inactivation accompanied by the epigenetic mark of H3K9me3. These results suggest that the "synapsis or silence model" may not be universal among animals.

\section{Materials and methods}

Adult males of Graphosoma italicum and Carpocoris fuscispinus (Heteroptera: Pentatomidae) collected in natural populations, under the appropriated licenses and conditions, from Madrid (Spain) were used for this study. The chromosome complement of both species is composed of 12 autosomes and two sex chromosomes (XX \& $/ \mathrm{XY}$ §) (Rebagliati et al., 2005 ).

\section{Slides preparation: spreading and squashing of spermatocytes}

Testes were removed, cleaned in PBS (137 mM NaCl, $\left.2.7 \mathrm{mM} \mathrm{KCl}, 10.1 \mathrm{mM} \mathrm{Na}_{2} \mathrm{HPO}_{4}, 1.7 \mathrm{mM} \mathrm{KH}_{2} \mathrm{PO}_{4}, \mathrm{pH} 7.4\right)$, and seminiferous tubules then processed for either spreading or squashing. For spreading of spermatocytes, we followed a drying-down technique based on that previously described (Peters et al., 1997) with slight modifications (Viera et al., $2009 \mathrm{~b}$ ). Briefly, the proximal region of tubules was dissected with an entomological pin and removed in order to eliminate mature spermatids and spermatozoa. Seminiferous tubules were homogenised with tweezers in $100 \mathrm{mM}$ sucrose in distilled water and macerated for $15 \mathrm{~min}$ at room temperature. Spermatocytes were simultaneously spread onto a clean slide and fixed with $1 \%$ paraformaldehyde in distilled water containing $0.15 \%$ Triton X-100. Slides were left to dry for $2 \mathrm{~h}$ in a moist chamber and finally washed with $0.08 \%$ Photo-Flo (Kodak) in distilled water and air-dried. For squashing, we followed the technique previously described (Page et al., 1998 ; Parra et al., 2002 ). Briefly, the testes were fixed in freshly prepared $2 \%$ formaldehyde in PBS containing $0.1 \%$ Triton X-100 (Sigma, St. Louis, Missouri, USA). After $5 \mathrm{~min}$, the proximal regions of tubules were placed on a slide previously coated with $1 \mathrm{mg} / \mathrm{ml}$ poly-L-lysine (Sigma) with a drop of fixative, and the tubules were gently minced with tweezers. The tubules were then squashed, the coverslip removed after freezing in liquid nitrogen and slides were immediately placed in PBS.

Immunofluorescence microscopy 
After fixation, spread and squashed preparations were rinsed three times for $5 \mathrm{~min}$ in PBS and incubated for $45 \mathrm{~min}$ at room temperature or overnight at $4{ }^{\circ} \mathrm{C}$ with the corresponding primary antibodies diluted in PBS. To detect the cohesin subunit SMC3, we employed either a polyclonal rabbit anti-SMC3 antibody (AB3914; Chemicon International) raised against a synthetic peptide from human SMC3, or the K987 rabbit polyclonal antisera (kindly provided by Dr. Barbero; (Viera et al., 2004a), raised against a synthetic peptide corresponding to the carboxy-terminal amino-acid sequence of human SMC3, both of them at 1:30 dilution. The rabbit polyclonal antisera K988 raised against a synthetic peptide corresponding to the carboxy-terminal amino-acid sequence of human SMC1 $\alpha$ (kindly provided by Dr. Barbero) was used to detect SMC1 $\alpha$ (Viera et al., 2009b ) at 1:30 dilution. The presence of RNA polymerase II was revealed with a mouse monoclonal antibody anti-RNA polymerase II phosphorylated at serine 2 (Abcam 24758) at 1:100 dilution. Histone $\mathrm{H} 3$ trimethylated at lysine 9 (H3K9me3) was revealed with a rabbit polyclonal serum (Abcam, ab-8898) at 1:100 dilution. A rabbit polyclonal anti-RAD51 antibody (Oncogene Research Products, Ab-1, PC130), generated against recombinant HsRad51 protein, was used at 1:50 dilution. Following three washes in PBS, the slides were incubated for $30 \mathrm{~min}$ at room temperature with secondary antibodies. The secondary antibodies used were donkey anti-rabbit $\operatorname{IgG}$ (Jackson) at 1:150 dilution and goat anti-mouse IgG (Jackson) at 1:150 dilution, both of them were conjugated with Texas Red or fluorescein isothiocyanate (FITC); and a donkey anti-mouse IgM conjugated with DyLight 594 (Jackson) to reveal RNA polymerase II antibody. The slides were subsequently rinsed in PBS, and chromatin was counterstained with $5 \mu \mathrm{g} / \mathrm{ml}$ DAPI (4', 6-diamidino-2-phenylindole) for $3 \mathrm{~min}$. After rinsing in PBS, the slides were mounted in Vectashield (Vector Laboratories, Burlingame, California, USA) and sealed with nail varnish. For the doubleimmunolabelling experiments in which two primary antibodies were generated in the same host species (SMC3 or SMC1 $\alpha$ with either RAD51 and H3K9tri-m), we proceed as previously described (Page et al., 2003 ; Viera et al., 2003 ): the slides were first incubated with the anti-SMC3 or anti-SMC1 $\alpha$ antibodies for $1 \mathrm{~h}$ at room temperature, rinsed three times for $5 \mathrm{~min}$ in PBS and incubated overnight at $4{ }^{\circ} \mathrm{C}$ with an DyLight 488-conjugated goat Fab' fragment anti-rabbit IgG (Jackson) at 1:100 dilution in PBS. Afterwards, slides were rinsed six times for $5 \mathrm{~min}$ in PBS, incubated with anti-RAD51 or anti-H3K9me3 for $1 \mathrm{~h}$, rinsed three times for $5 \mathrm{~min}$ in PBS, and then incubated with a donkey anti-rabbit IgG (Jackson) at 1:150 dilution. In the case of triple immunolocalisation of SMC3, H3K9tri-m and RNA polymerase II the slides were first incubated for $1 \mathrm{~h}$ with anti-SMC3 antibody, rinsed in PBS and incubated overnight at $4{ }^{\circ} \mathrm{C}$ with a 
DyLight-488 conjugated Fab' fragment goat anti-rabbit IgG (Jackson) at 1:100 dilution. After rinsing six times for 5 min in PBS, the slides were incubated simultaneously with antibodies against H3K9tri-m and against RNA polymerase II for $1 \mathrm{~h}$, rinsed three times for $5 \mathrm{~min}$ in PBS and then incubated with donkey anti-mouse IgM conjugated with DyLight 594 (Jackson) at 1:150 dilution and with DyLight 405-conjugated donkey anti-rabbit IgG (Jackson) at 1:150 dilution.

Observations were performed using an Olympus BX61 microscope (Olympus, Hamburg, Germany) equipped with motorised $\mathrm{Z}$ axis and epifluorescence. Single images or image stacks were captured with an Olympus DP71 digital camera controlled by CellF Imaging System (Münster, Germany) under standardised capture conditions in order to facilitate the comparison of signals intensity among different meiotic stages. Images were analysed and processed using the public domain ImageJ software (National Institutes of Health, USA; http://rsb.info.nih.gov/ij). Final images were processed with Adobe Photoshop 7.0 software.

\section{Results}

In order to precisely determine the achievement of synapsis, and hence the meiotic stage of prophase I spermatocytes, we analysed the distribution of SMC1 $\alpha$ and SMC3, two members of the structural maintenance of chromosomes (SMC) family of proteins, that are widely conserved in eukaryotes (Hirano, 2002 ). These two proteins are subjacent to the axial and lateral elements of the synaptonemal complex in mammals (Eijpe et al., 2000 ; James et al., 2002 ; Pelttari et al., 2001 ). Consequently, the pairing progression of SMC1 $\alpha / 3$ axes during prophase I reflects the synaptic state of bivalents and represents a reliable tool for the analyses of species in which antibodies against components of the synaptonemal complex are not available. Since both cohesin subunits produced an identical pattern of distribution during meiosis in both species (G. italicum and C. fuscispinus), hereon, we will only refer to the results obtained for SMC3 in order to avoid redundancy. Staging of meiotic prophase was performed on the basis of cohesin axes pairing and the appearance of recombination markers, such as RAD51. A minimum of 50 spermatocytes were scored for each stage and immunolabelling combination. The discrimination between zygotene and diplotene spermatocytes was particularly important. In this case, we have followed previously validated criteria (Viera et al., 2009a): (i) SMC3 axes are recognisable in zygotene, but not in diplotene, as it is preceded by the diffuse stage in which cohesin axes disorganize, 
(ii) RAD51 foci can be seen in zygotene nuclei, when meiotic recombination is active, but not in diplotene, and (iii) chromosomes are not individualised from leptotene up to the diffuse stage, but individual bivalents and the sex chromosomes are clearly discerned in diplotene.

\section{Transcriptional activity in G. italicum spermatocytes}

In order to determine the transcriptional activity of G. italicum spermatocytes, we performed a double immunolabelling of SMC3 and phosphorylated RNA polymerase II (pRNApol II). It must be noted that, as we have previously reported, the anti-SMC3 antibody labelled the cohesin axes and occasionally the nucleolus (Viera et al., 2009a). In early leptotene spermatocytes, single unpaired cohesin axes were formed along chromosomes, while pRNApol II labelling was not evident in the nucleus (Fig. 1a-d ). During zygotene, SMC3 axes started to pair giving rise to thicker SMC3 filaments as a consequence of homologous chromosomes synapsis progression and pRNApol II signal became observable dispersed over the nucleus (Fig. 1e-h ). In no case, association of pRNApol II signal was specific of regions where chromosomes have achieved synapsis. This indicates that the active or inactive state of chromosomes/chromosome domains does not depend on their synaptic state. By early pachytene, autosomes achieved complete synapsis and a single thick cohesin SMC3 axis per bivalent, corresponding to the paired axes of both homologues, was seen. Additionally, the SMC3 axes corresponding to the $\mathrm{X}$ and $\mathrm{Y}$ chromosomes appeared single and unpaired and were usually located at the nuclear periphery (Fig. 1i, j ). Labelling of pRNApol II was highly increased if compared with previous stages and it fully covered the chromatin of the autosomes, but was absent in the sex chromosomes (Fig. 1k, 1). This transcriptional pattern was maintained almost invariable during late pachytene (when SMC3 axes start to disassemble and become discontinuous and fragmented) (Fig. 1m-p), the diffuse stage (with disorganised cohesin axes) (Fig. 1q-t), and diplotene (Fig. 1u-x ). Thus, in G. italicum spermatocytes, transcriptional activity was either low or undetectable at the onset of meiosis, gradually increased during zygotene and reached a high level during pachytene and later stages of the first meiotic prophase. Thereafter, pRNApol II labelling decreased and was undetectable at metaphase I and subsequent stages up to telophase II (Supplementary video 1 ).

Fig. 1 
Double immunolabelling of SMC3 (green) and pRNApol II (blue) in spread prophase I spermatocytes of G. italicum, counterstained with DAPI (grey). The position of the sex chromosomes is indicated $(X Y)$ in $\mathbf{i}-\mathbf{x}$ and sex chromatin has been outlined in red in $\mathbf{j}-\mathbf{l}$ for their localisation. a-d Leptotene. Stretches of maturing SMC3 axes appear dispersed in the nucleus and no signal of pRNApol II is detectable. $\mathbf{e}-\mathbf{h}$ During zygotene, the thin SMC3 axes of homologues (arrows in $\mathbf{e}$ ) start to pair into thicker filaments (arrowheads in e) and pRNApol II faintly labels the chromatin. i-l) Fully paired SMC3 axes are evident at autosomes in pachytene (arrowheads in i), while single and thin unpaired axes (arrows in i) are present in the sex chromosomes ( $X Y$; outlined in red in $\mathbf{j}$. pRNApol II covers the chromatin of autosomes, but is absent in the sex chromosomes $(X Y$, outlined in red in $\mathbf{k}, \mathbf{l})$. $\mathbf{m}-\mathbf{p}$ By late pachytene, SMC3 axes start to disorganize, but a SMC3 accumulation at the nucleolus $(\mathrm{Nu})$ can be found. In this stage, pRNApol II remains associated to the autosomal chromatin, but is absent in the sex chromosomes $(X Y)$. q-t The diffuse stage is characterised by the complete disorganisation of SMC3 axes. An intense pRNApol II staining covers the chromatin, except at the region occupied by the sex chromosomes $(X Y)$. u$\mathbf{x}$ In diplotene, desynapsed and individualised autosomal bivalents are detected after DAPI staining. SMC3 is not detectable, but an extensive pRNApol II labelling is present in the bivalents, except in the sex chromosomes $(X Y)$ 


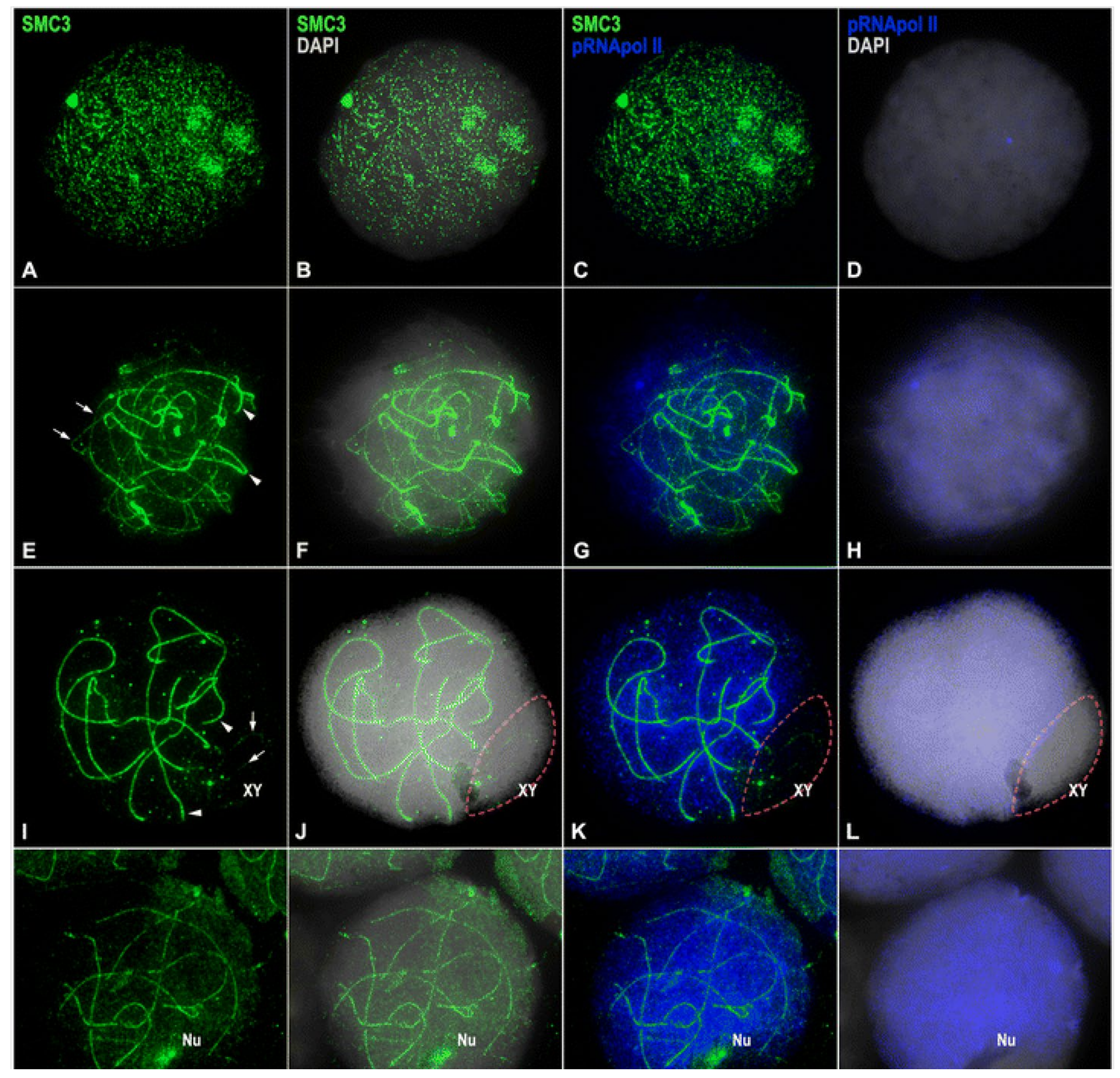


In order to explore the presence of epigenetic modifications related to transcriptional regulation, we first tried to localise $\gamma \mathrm{H} 2 \mathrm{AX}$. However, two different antibodies raised against the human protein and an antibody against its Drosophila homologue H2Av (gently provided by Dr. Kim McKim) did not give any positive result (not shown). H3K9me3, an epigenetic mark usually related to heterochromatin formation (Cowell et al., 2002 ) was then immunolocalised together with SMC3 and pRNApol II. During leptotene and early zygotene, the absence or low level of pRNApol II was accompanied by low labelling of H3K9me3 which, in no case, covered most of the chromatin (Fig. 2a, b ). At mid zygotene, pRNApol II had a weak signal in the entire nucleus (Fig. 2c, d ), while H3K9me3 labelling was more concentrated at specific nuclear regions, of different sizes and intensity, where the SMC3-labelled cohesin axes ends were located (Fig. 2c, d ). Throughout pachytene, the intensity of pRNApol II labelling was highly increased and almost covered the whole nucleus, excepting the sex chromosomes (Fig. 2e and g), despite the spreading procedure might cause some overlapping. Concurrently, H3K9me3 accumulations were observed, under our standardised capture conditions, at the ends of most of the autosomal bivalents and the chromatin of the sex chromosomes (Fig. $2 \mathrm{f}$ and $\mathrm{h}$ ). It must be pointed out that, although we have previously reported the accumulation of H3K9me3 at every end of the SMC3 axes (Viera et al., 2009a; Viera et al., 2009c), in the present study, the capture conditions were standardised for allowing the comparison of intensities in the different meiotic stages. Thus, in some cases, the H3K9me3 accumulation is not seen at certain SMC3 axis ends due to the low intensity of the signal.

\section{Fig. 2}

Triple immunolabelling of SMC3 (green), pRNApol II (blue) and H3K9me3 (red) in spread spermatocytes of G. italicum (aj) and double immunolabelling of pRNApol II (blue) and H3K9me3 (red) in squashed spermatocytes of G. italicum (k, l). The position of the sex chromosomes is indicated $(X Y)$ in $\mathbf{e}-\mathbf{l}$. DAPI staining (grey) is shown in $\mathbf{k}, \mathbf{l} . \mathbf{a}, \mathbf{b}$ Early leptotene spermatocytes display low levels of pRNApol II and H3K9me3 as SMC3 axes are forming. $\mathbf{c}$, d During zygotene, as SMC3 axes start to pair, the intensity of pRNApol II and H3K9me3 labelling increases. e, f Pachytene spermatocyte: pRNApol II is absent from the univalent sex chromosomes $(X Y)$, but is spread over all the chromatin of autosomes. H3K9me3 appears restricted to the tips of the fully paired SMC3 axes of autosomes and covers the chromatin of the sex chromosomes $(X Y)$. g, h As SMC3 axes disorganize in late pachytene, pRNApol II and H3K9me3 maintain their locations. $\mathbf{i}$, $\mathbf{j}$ In diffuse stage 
nuclei, SMC3 axes are no longer observable, but the relative distribution of pRNApol II and H3K9me3 remain unaltered. $\mathbf{k}$, I Diplotene desynapsing autosomal bivalents are recognisable after DAPI staining. pRNApol II distributes over the entire nucleus, except in the chromatin of the sex chromosomes $(X Y)$, while H3K9me3 labels the ends of the autosomal bivalents and covers the sex chromosomes $(X Y)$ 


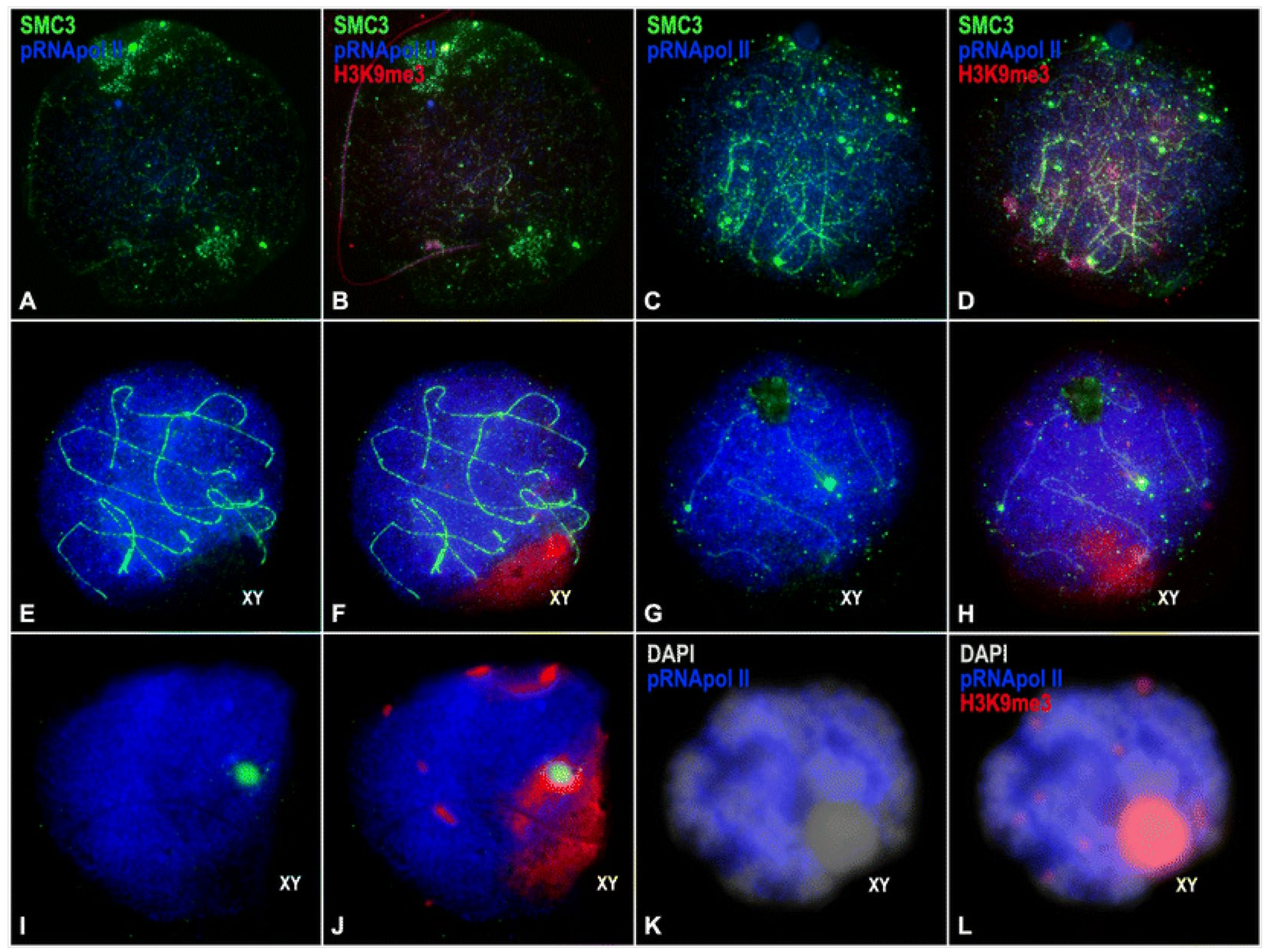

At the diffuse stage, the labelling intensity and distribution of both pRNApol II and H3K9me3 at the nucleus were 
maintained, albeit SMC3 axes were disorganised (Fig. 2i, j). Diplotene nuclei showed pRNApol II signals covering the whole nucleus excepting the sex chromosomes (Fig. 2k ), whereas H3K9me3 labelling appeared as spherical signals located at the chromosome ends and was highly accumulated on the sex chromosomes (Fig. 21 ; Supplementary video 2 ). Thus, our data demonstrated that the blurry $\mathrm{H} 3 \mathrm{~K} 9 \mathrm{me} 3$ labelling found in leptotene gradually became detectable as small discrete regions at the ends of synapsed autosomal bivalents and as a huge and intense mass at the sex chromosomes (Fig. 2 ).

Taking into account the distribution of $\mathrm{H} 3 \mathrm{~K} 9 \mathrm{me} 3$ in G. italicum spermatocytes, and that the spreading procedure disperses chromatin, we wondered whether $\mathrm{H} 3$ was specifically modified at different chromatin domains during prophase I, or remained invariably associated to the autosome ends and the sex chromosomes. For this purpose, we performed double immunolabelling of SMC3 and H3K9me3 in squashed preparations (Fig. 3 ), since this technique does not alter chromatin condensation and facilitates the identification of specific nuclear regions. During leptotene, H3K9me3 faintly labelled the chromatin and was restricted to discrete domains of the periphery of the nucleus (Fig. 3a-d ) where the ends of SMC3 axes associated to the nuclear envelope were located (Fig. 3a-d ). Additionally, a large faint accumulation of H3K9me3 was detected in these spermatocytes (Fig. 3c, d ). Due to the presence of extensive unpaired chromosomal regions, we were not able to determine whether this structure corresponded to any specific chromosome (Fig. 3a, b ). A similar H3K9me3 accumulation was found in zygotene (Fig. 3a). A detailed analysis of these nuclei showed that the H3K9me3 mass was invariably associated to the unpaired SMC3 axes of the sex chromosomes (Fig. 3a and c). In pachytene, SMC3 axes were fully paired in the autosomes, but remained single and unpaired in the sex chromosomes (Fig. 3a, b ). Interestingly, H3K9me3 was mainly found at the ends of the paired SMC3 axes, located at the nuclear periphery, and covered the chromatin of the sex chromosomes (Fig. 3a-d ). It is noteworthy that this distribution of H3K9me3 is maintained throughout prophase I (Supplementary video 3 ) as shown in spread spermatocytes (Fig. 2 ). In order to ascertain whether the ends and the sex chromosomes were covered by H3K9me3 at the beginning of meiosis, we analysed the distribution of $\mathrm{H} 3 \mathrm{~K} 9 \mathrm{me} 3$ in spermatogonial cells. The identification of prometaphase spermatogonial cells was simple and unambiguous after DAPI-staining since 14 condensing chromosomes (12 autosomes + XY) were found. In these cells, the chromatin of both $\mathrm{X}$ and $\mathrm{Y}$ chromosomes, recognisable as the longest and the shortest chromosomes 
respectively, was intensively labelled by H3K9me3 (Fig. 3e-g; Supplementary Fig. 1 and Supplementary video 4 ).

Moreover, autosomal chromosome ends also presented discrete $\mathrm{H} 3 \mathrm{~K} 9 \mathrm{me} 3$ accumulations, although the entire chromatin was faintly labelled (Fig. 3e-g; Supplementary Fig. 1 and Supplementary video 4 ). These results strongly indicate that H3K9me3 represents a constitutive epigenetic modification of these chromatin domains.

\section{Fig. 3}

Double immunolabelling of SMC3 (green) and H3K9me3 (red) in squashed prophase I spermatocytes and squashed spermatogonial cells of $G$. italicum. The position of the sex chromosomes is indicated $(X Y)$ in $\mathbf{b}, \mathbf{d}$ and $\mathbf{g}$. DAPI staining (blue) is shown in $\mathbf{b}, \mathbf{d}$, e and $\mathbf{g}$. The image corresponds to the superposition of 25 focal planes across the cells. a, b Leptotene $(L e)$, zygotene $(Z y)$ and pachytene $(P a)$ spermatocytes. a The unpaired SMC3 axes found in leptotene gradually pair during zygotene until they appear fully paired in the autosomes of pachytene spermatocytes. $\mathbf{b}$ In these spermatocytes, the chromatin of the sex chromosomes $(X Y)$ is only clearly identified in pachytene spermatocytes. $\mathbf{c}, \mathbf{d}$ During leptotene and up to pachytene, H3K9me3 shows faint labelling over the chromatin and is highly enriched at discrete domains (arrows) where SMC3 axes end, and in large masses (asterisks) that cover the sex chromosomes (XY). e-g Spermatogonial prometaphase cells. These cells are recognised after DAPI-staining by the presence of 14 condensing chromosomes. Notice that H3K9me3 is mostly detected at discrete domains of the chromosome ends (arrows) and at large accumulations (asterisks) covering the chromatin of the sex chromosomes $(X Y)$ 


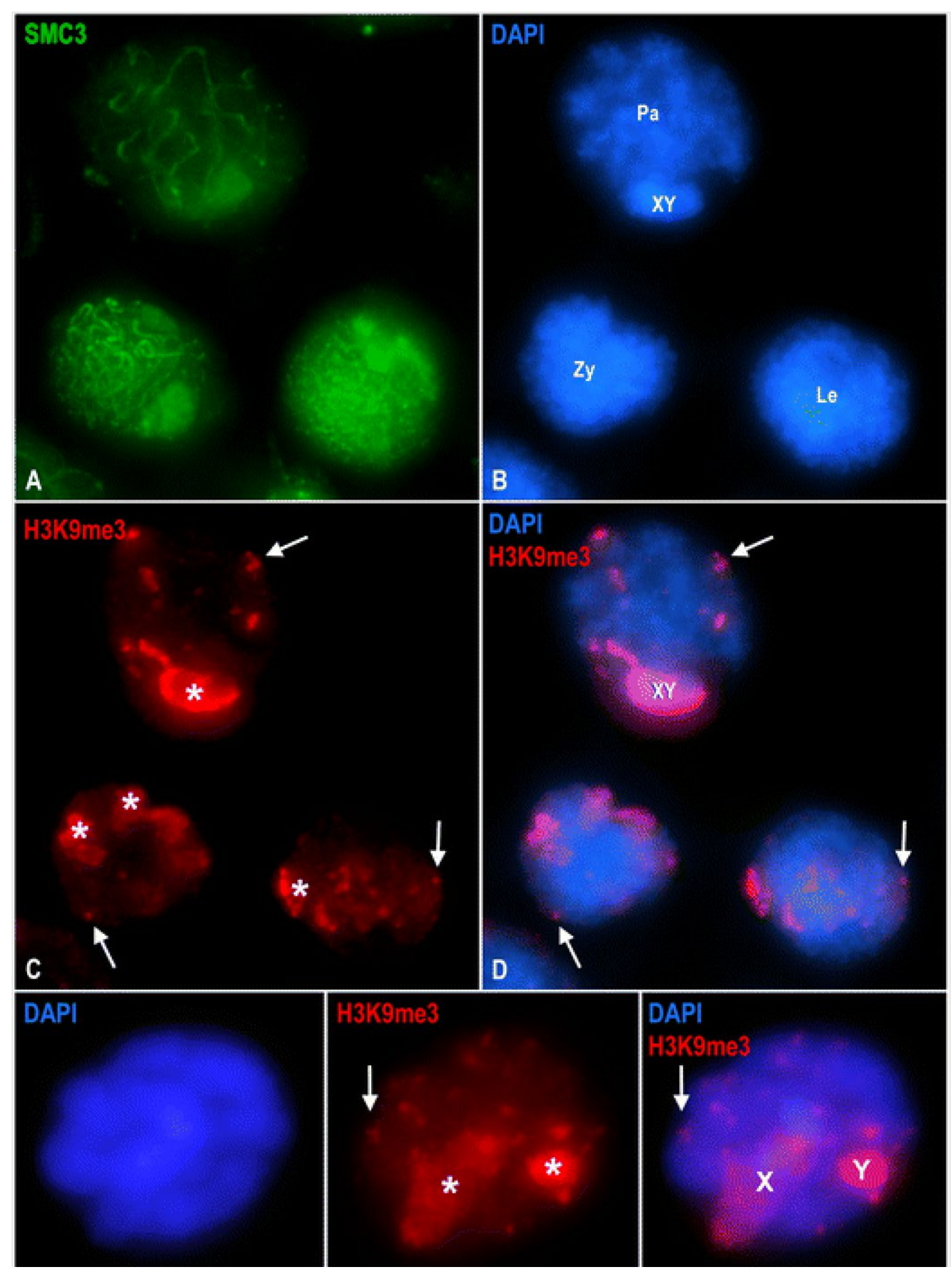




\section{Chromosome pairing and recombination initiation in C. fuscispinus spermatocytes}

We then carried out a similar study in another bug, C. fuscispinus, that has the same karyotype and sex chromosome determinism to those of G. italicum. However, both species clearly differ in the timing and the morphological achievement of synapsis. Therefore, a precise identification of the meiotic stages and the progression of the meiotic process was necessary. As previously reported (Viera et al., 2009a), the double immunolabelling of the cohesin subunit SMC3 and RAD51 together with chromatin morphology are reliable tools for establishing an accurate and unambiguous identification of all prophase I stages in hemipterans. Consequently, we performed a double immunolabelling of SMC3 and RAD51 in C. fuscispinus spermatocytes, prior to establishing the transcriptional activity in this species.

C. fuscispinus leptotene spermatocytes showed discrete stretches of SMC3 axes over the entire nucleus (Fig. 4a), while an abundant RAD51 labelling was not observed (Fig. 4b ). As synapsis initiates in early zygotene, the SMC3 axes of homologous chromosomes started to associate at a certain nuclear region corresponding to the basis of the bouquet-like arrangement of chromosomes (Fig. 4c). Nonetheless, a detailed analysis demonstrated that the association of SMC3 axes did not occur as in G. italicum that shows thin homologous SMC3 axes intimately associated into continuous thicker filaments which gradually expand along the homologues (Viera et al., 2009a; Figs. 1 and 2 ). By contrast, in $C$. fuscispinus, homologous SMC3 axes run in parallel aligned to each other but remained slightly separated, appearing only in contact at several discrete points along their length (Fig. $4 \mathrm{c}$ and d'). This phenomenon gives rise to bubbles along the SMC3 axes of homologues (Fig. 4c and d '). In this species, one can assume that the onset of synapsis initiates at chromosome ends, since the tips of the SMC3 axes were the first aligned regions (Fig. 4c). In these spermatocytes, several RAD51 foci were located along SMC3 axes (Fig. $4 \mathrm{~d}$ and d'). During mid and late zygotene, the length of aligned SMC3 axes increased, until they reached the entire length of autosomal bivalents. Moreover, numerous bubbles were seen along those axes (Fig. 4e, g, $\mathrm{f}^{\prime}$ and h'), indicating that synapsis is achieved at the same time at several locations of the homologues. Since the sex chromosomes are asynaptic, they were recognisable for the presence of a single thin and unaligned SMC3 axis (Fig. 4g). The massive RAD51 labelling shown in mid zygotene as foci located along SMC3 axes (Fig. 4f and f') was progressively reduced up to late zygotene (Fig. 4h and h '). Interestingly, RAD51 was absent from the SMC3 axes of the sex chromosomes (Fig. 4h ). In pachytene, the synapsed autosomal bivalents 
showed thick and fully paired SMC3 axes, whereas the asynaptic $\mathrm{X}$ and $\mathrm{Y}$ chromosomes had individual and thin axes (Fig. 4i and i'; see also Fig. 5 ). It must be noted that the bubbles along SMC3 axes were not observed in pachytene (Fig. $4 \mathrm{i}$ and $\mathrm{j}$ '), whereas some RAD51 foci were still observed along the paired SMC3 axes of the autosomal bivalents (Fig. $4 \mathrm{j}$ and $\mathrm{j}$ '). By late pachytene, the SMC3 axes started to disorganize and appeared as irregular and discontinuous filaments at the nuclei, SMC3 accumulated at a discrete nuclear region that probably corresponds to the nucleolus (as observed in G. italicum) (Fig. 4k ) and RAD51 foci were not observable over the disassembling SMC3 axes (Fig. 41 and 1 '). The diffuse stage, in which the SC disassembles, was characterised by the absence of SMC3 filaments (although the antibody labelled the nucleolus), the absence of RAD51 foci and the blurry appearance of the chromatin (Fig. 4m, $\mathrm{n}$ ). During diplotene, SMC3 was only detectable at the nucleolus and RAD51 remained absent in the nucleus (Fig. 4o, p). Neither SMC3 nor RAD51 were detected in further meiotic stages (not shown).

\section{Fig. 4}


Double immunolabelling of SMC3 (green) and RAD51 (red) in spread prophase I spermatocytes of C. fuscispinus. The position of the sex chromosomes is indicated $(X Y)$ in $\mathbf{g}-\mathbf{j}$ and $\mathbf{m}-\mathbf{p} . \mathbf{a}, \mathbf{b}$ In leptotene, SMC3 axes appear in the entire nucleus, while RAD51 foci are almost undetectable. $\mathbf{c}, \mathbf{d}$ and $\mathbf{d}$ ' During early zygotene, homologous SMC3 axes start to run in parallel and contacts along them give rise to bubbles (arrows in d'). Additionally, numerous RAD51 foci locate over SMC3 axes. e, f and f' Mid zygotene spermatocyte. The progressive alignment of SMC3 axes continues along homologous chromosomes showing bubbles along them (arrows in f') and RAD51 foci over the cohesin axes. $\mathbf{g}$, $\mathbf{h}$ and $\mathbf{h}$ ' By late zygotene SMC3 axes are aligned at the entire length of the autosomal bivalents, while remain unpaired at the asynaptic sex chromosomes $(X Y)$. RAD51 foci locate exclusively at the autosomal SMC3 axes (h'). $\mathbf{i}, \mathbf{i}, \mathbf{j}$ and $\mathbf{j}$ ' Pachytene spermatocyte. Autosomal bivalents display fully paired and thick SMC3 axes (arrows in $\mathbf{i}$ and $\mathbf{i}$ ) with few RAD51 foci along them (j'). The asynaptic sex chromosomes (XY) display a single unpaired SMC3 axis (arrowheads in $\mathbf{i}$ and $\mathbf{i}$ ) in their chromatin (grey in $\mathbf{i}$ ). $\mathbf{k}, \mathbf{l}$ and $\mathbf{l}$ ' Late pachytene nucleus showing the disassembly of SMC3 axes, which become discontinuous, and the dramatic decrease of RAD51 foci (l'). $\mathbf{m}, \mathbf{n}$ Diffuse stage spermatocyte. SMC3 only remains visible as an accumulation close to the chromatin of the sex chromosomes $(X Y)$ (grey in $\mathbf{n}$ ), which likely represents the nucleolus $(N u)$. RAD51 foci are no longer detectable. o, p During diplotene, bivalents start to desynapse and individualise. The SMC3 accumulation at the nucleolus $(N u)$ persists close to the sex chromosomes $(X Y)$. q C. fuscispinus adult male 


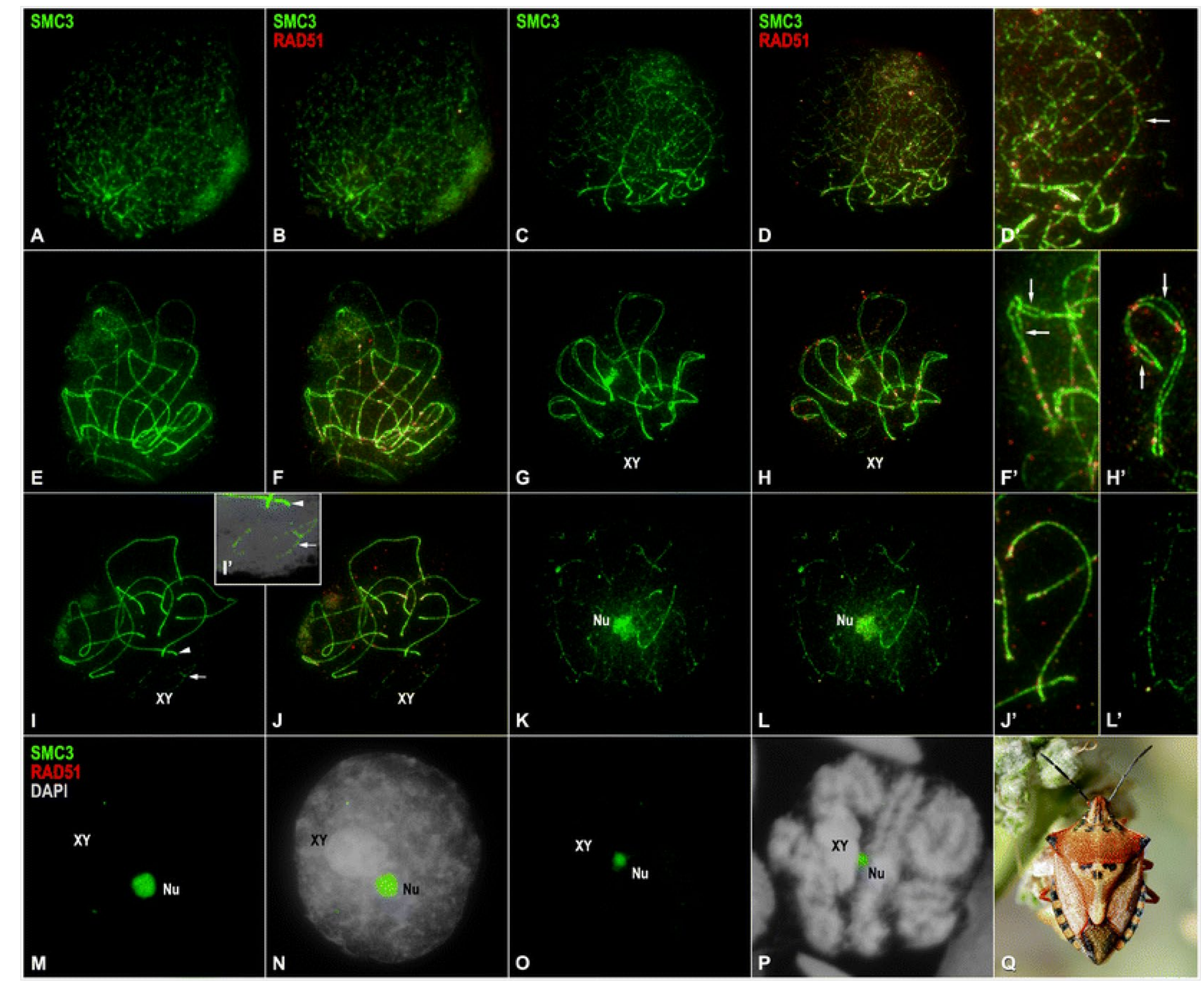


Fig. 5

Double immunolabelling of SMC3 (green) and pRNApol II (blue) in spread prophase I spermatocytes of C. fuscispinus, counterstained with DAPI (grey). The position of the sex chromosomes is indicated $(X Y)$ in $\mathbf{i}-\mathbf{x}$ and the sex chromatin has been outlined in red in $\mathbf{j}-\mathbf{l}$ for their localisation. a-d In leptotene spermatocytes, pRNApol II faintly labels the nucleus as SMC3 axes are forming. $\mathbf{e}-\mathbf{h}$ During zygotene, SMC3 axes of autosomal bivalents get aligned at their entire length with pRNApol II intensively covering their chromatin. By contrast, a complete absence of pRNApol II labelling is found over a nuclear region where single unpaired SMC3 are present. i-l Pachytene spermatocyte: SMC3 axes of autosomes have achieved complete pairing into thicker filaments (arrowheads in i) but those of the sex chromosomes remain thin and unpaired (arrows in i) ( $X Y$; outlined in red in $\mathbf{j}$ ). A massive pRNApol II staining spreads over the chromatin, except that of the sex chromosomes ( $X Y$; outlined in red in $\mathbf{k}, \mathbf{l})$. $\mathbf{m}-\mathbf{p}$ At the end of pachytene, SMC3 axes begin to disassemble, but an SMC3 accumulation is observed at the nucleolus $(N u)$. pRNApol II labelling persists covering the autosomal chromatin, but is absent in the sex chromosomes $(X Y)$. q-t Diffuse stage spermatocyte: SMC3 axes are undetectable while the intense pRNApol II staining is absent in the chromatin of the sex chromosomes $(X Y)$. u-x In diplotene, pRNApol II labelling continues associated to the autosomal bivalents and is absent in the sex chromosomes $(X Y)$ 


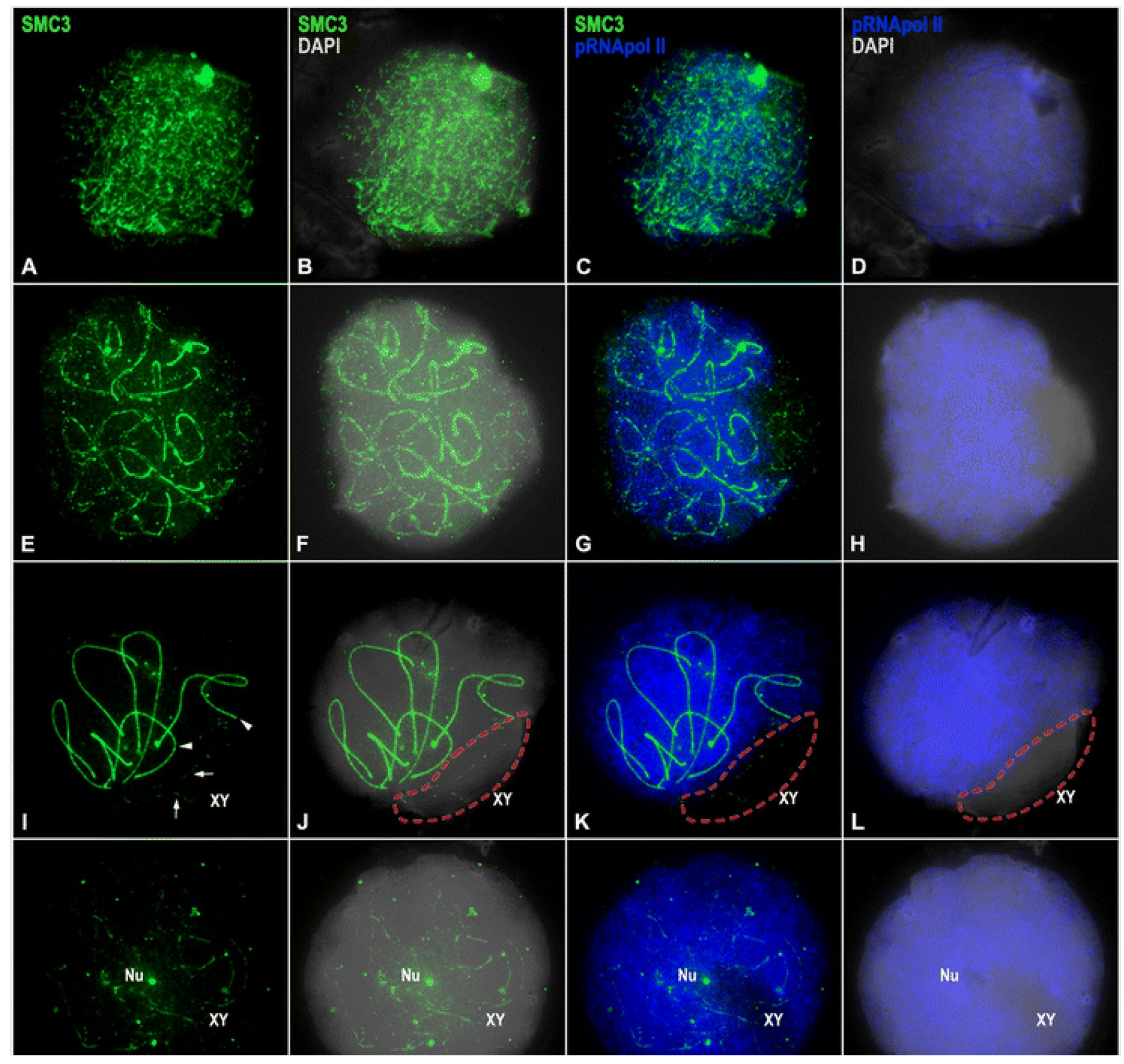




\section{Transcriptional activity in C. fuscispinus spermatocytes}

Since transcriptional activity has been related to synapsis progression during prophase I, and taking into account the characteristic pattern of synapsis progression found in C. fuscispinus, we tested the relationship between synapsis and transcription in this species by the double immunolocalisation of SMC3 and pRNApol II. During the maturation of SMC3 axes, in leptotene spermatocytes, a faint pRNApol II labelling was observed over most of the nucleus (Fig. 5a-d ). Throughout zygotene, pRNApol II labelling increased and covered almost all the chromatin (Fig. 5e-h ). Thus, pRNApol II was located at regions displaying both unpaired and aligned SMC3 axes of the autosomal bivalents (Fig. 5g). Interestingly, it was also evident the absence of pRNApol II in the unpaired SMC3 axis corresponding to the sex chromosomes (Fig. $5 \mathrm{~g}, \mathrm{~h}$ ). At the onset of pachytene, the achievement of synapsis in autosomal bivalents, shown as fully paired thick SMC3 axes, was accompanied by an increase in the intensity of pRNApol II labelling (Fig. 5i-1 ). pRNApol II covered all of the chromatin of autosomes but was absent in the sex chromosomes (Fig. 5i-1). The absence of any detectable pRNApol II signal on the sex chromosomes was maintained in late pachytene (SMC3 axes start to disorganize) (Fig. 5m-p), in the diffuse stage (no detectable SMC3 axes) (Fig. 5q-t), and in diplotene (Fig. 5u-x ). As described for G. italicum, pRNApol II was undetectable from metaphase I up to telophase II (not shown).

Subsequently, we determined the localisation of H3K9me3 in relation to SMC3 (as occurred in G. italicum, we have not observed any labelling with antibodies against $\gamma \mathrm{H} 2 \mathrm{AX}$ or $\mathrm{H} 2 \mathrm{Av}$ ). In this case, H3K9me3 appeared in some nuclear regions during leptotene and early zygotene (Fig. 6a-d ). Interestingly, these regions may involve chromosomal domains with both unpaired and aligned SMC3 axes (Fig. 6a-d ). During mid to late zygotene, the H3K9me3 labelling clearly covered the chromatin of the asynaptic sex chromosomes (Fig. 6e-h ). In pachytene, the H3K9me3 labelling associated to the sex chromosomes was evident (Fig. 6i, j) and was maintained in late pachytene, when SMC3 axes start to disassemble (Fig. 6k, 1). Diffuse stage nuclei did not present SMC3 axes along the chromosomes, although this protein accumulated in a region corresponding to the nucleolus. Moreover, H3K9me3 was mainly associated with the sex chromosomes (Fig. 6m, n ), albeit some additional foci were also seen dispersed in the nucleus (Fig. 6n ). These foci may correspond to the autosomal ends as previously observed in G. italicum (Fig. 2 ; Viera et al., 2009a). In diplotene, H3K9me3 showed discrete signals located at the ends of desynapsing autosomal bivalents and covered all the chromatin 
of the sex chromosomes (Fig. 6o , p). This was also observed in G. italicum (Fig. 2 ; Supplementary video 2 ; Viera et al. 2009a).

\section{Fig. 6}

Double immunolabelling of SMC3 (green) and H3K9me3 (red) in prophase I spread spermatocytes of C. fuscispinus. The position of the sex chromosomes is indicated $(X Y)$ in $\mathbf{e - p}$. DAPI staining (grey) is shown in $\mathbf{0}, \mathbf{p}$. a, b In leptotene spermatocytes, $\mathrm{H} 3 \mathrm{~K} 9 \mathrm{me} 3$ becomes increasingly visible as diffuse masses over some chromatin regions as SMC3 axes are forming. c-f During early and mid zygotene, the H3K9me3 labelling progressively concentrates at certain regions of autosomes and covers the chromatin of the sex chromosomes $(X Y)$. $\mathbf{g}, \mathbf{h}$ By late zygotene, H3K9me3 labelling is mainly restricted to the sex chromosomes $(X Y)$. i, j Pachytene spermatocyte. SMC3 axes are completely paired along the autosomal bivalents, but remain unpaired at the sex chromosomes $(X Y)$. The chromatin of the sex chromosomes appears profusely labelled by $\mathrm{H} 3 \mathrm{~K} 9 \mathrm{me} 3$. $\mathbf{k}$, I SMC3 axes disorganize by late pachytene, while H3K9me3 persists in the sex chromosomes $(X Y) . \mathbf{m}, \mathbf{n}$ In diffuse stage spermatocytes, SMC3 is no longer visible at chromosome axes, but appears as large aggregates. H3K9me3 spreads in the sex chromosomes $(X Y)$ and also concentrates at discrete dispersed regions of the nucleus. o, $\mathbf{p}$ During diplotene, the H3K9me3 labelling covers the sex chromosomes $(X Y)$ and discrete accumulations are recognisable at the autosome ends 


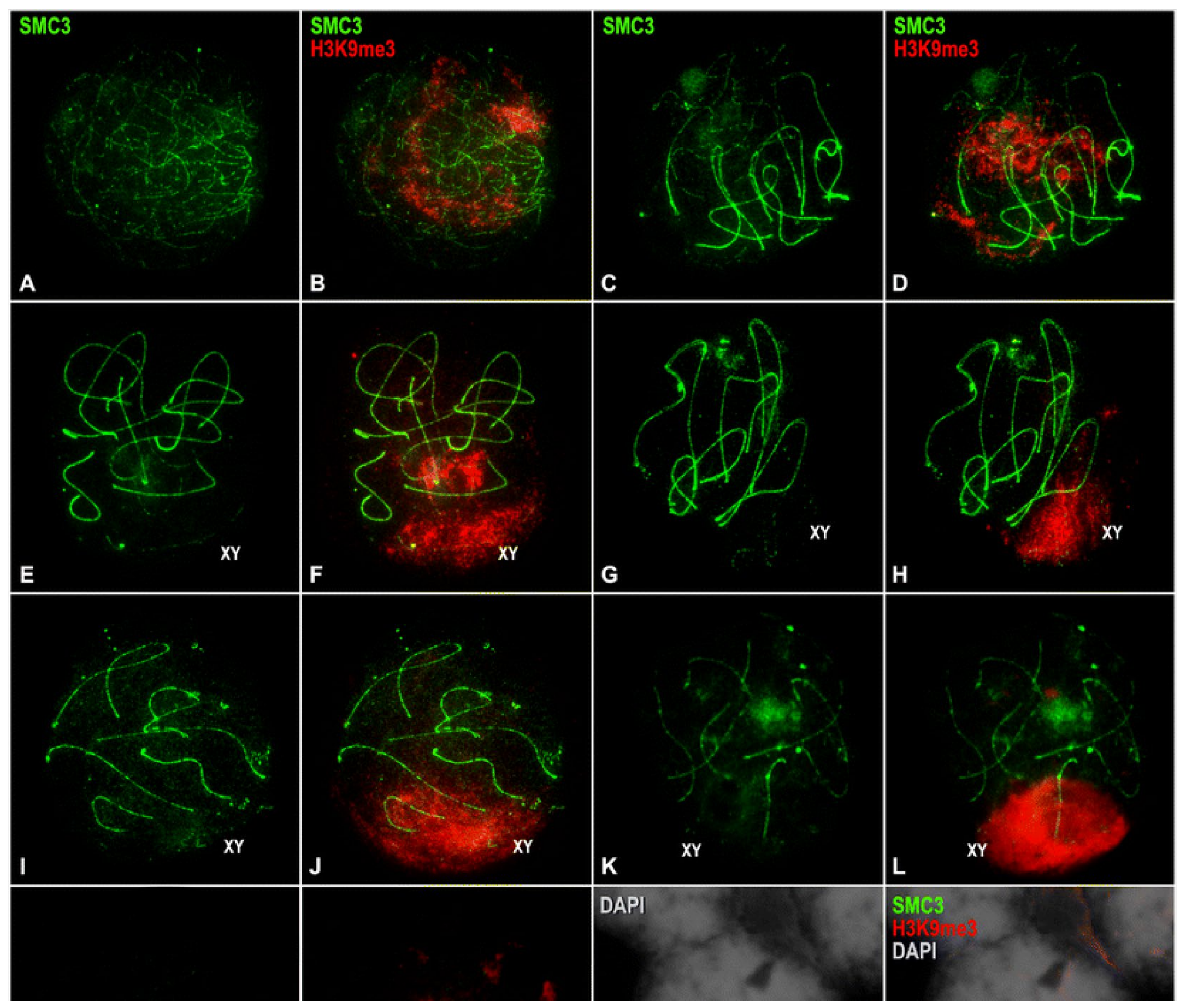


In order to correlate the transcriptional activity pattern and chromatin modification in C. fuscispinus spermatocytes, we performed a triple immunolabelling of SMC3, pRNApol II and H3K9me3 (Fig. 7 ). Our observations demonstrated low levels of pRNApol II and H3K9me3 during the formation of SMC3 axes in leptotene (Fig. 7a, b ). During the alignment of the SMC3 axes in zygotene, the intensity of both pRNApol II and H3K9me3 increased (Fig. 7c, d ). However, the distribution of these proteins was clearly segregated and almost mutually exclusive: while pRNApol II almost covered the entire nucleus, H3K9me3 was mainly restricted to the sex chromosomes (Fig. 7c, d). This differential labelling pattern was maintained throughout prophase I, independently of the maturation stage of SMC3 axes and the synaptic state of chromosomes (Fig. 7e-1).

\section{Fig. 7}

Triple immunolabelling of SMC3 (green), pRNApol (blue) and II H3K9me3 (red) in spread spermatocytes of C. fuscispinus (a-j) and double immunolabelling of pRNApol II (blue) and H3K9me3 (red) in squashed spermatocytes of C. fuscispinus $(\mathbf{k}, \mathbf{l})$. The position of the sex chromosomes is indicated $(X Y)$ in $\mathbf{c}-\mathbf{l}$. DAPI staining (grey) is shown in $\mathbf{k}, \mathbf{l}$. a, $\mathbf{b}$ The initial formation of SMC3 axes in early leptotene is accompanied by low levels of pRNApol II and H3K9me3. c, d Throughout zygotene, the SMC3 axes of autosomes align and the levels of pRNApol II and H3K9me3 increase. Interestingly, while pRNApol II covers the chromatin of autosomes, H3K9me3 concentrates in the asynaptic sex chromosomes $(X Y)$. e-h Pachytene spermatocytes with fully paired SMC3 axes in the autosomal bivalents and single unpaired axis in the sex chromosomes $(X Y)$. The distinctive distributions of pRNApol II in the autosomes and H3K9me3 in the sex chromosomes are maintained even when the SMC3 axes start to disorganize in late pachytene. $\mathbf{i}, \mathbf{j}$ The absence of SMC3 axes in diffuse stage spermatocytes is accompanied by pRNApol II profusely distributed in the chromatin of autosomes. H3K9me3 appears as some discrete aggregates dispersed in the nucleoplasm that do not correspond to the massive labelling of the sex chromosomes $(X Y) . \mathbf{k}, \mathbf{l}$ In diplotene spermatocytes, the desynapsing autosomal bivalents display massive pRNApol II labelling that is absent from the sex chromosomes $(X Y)$. By contrast, H3K9me3 locates as dots at the ends of the autosomal bivalents and spreads over the chromatin of the sex chromosome $(X Y)$ 


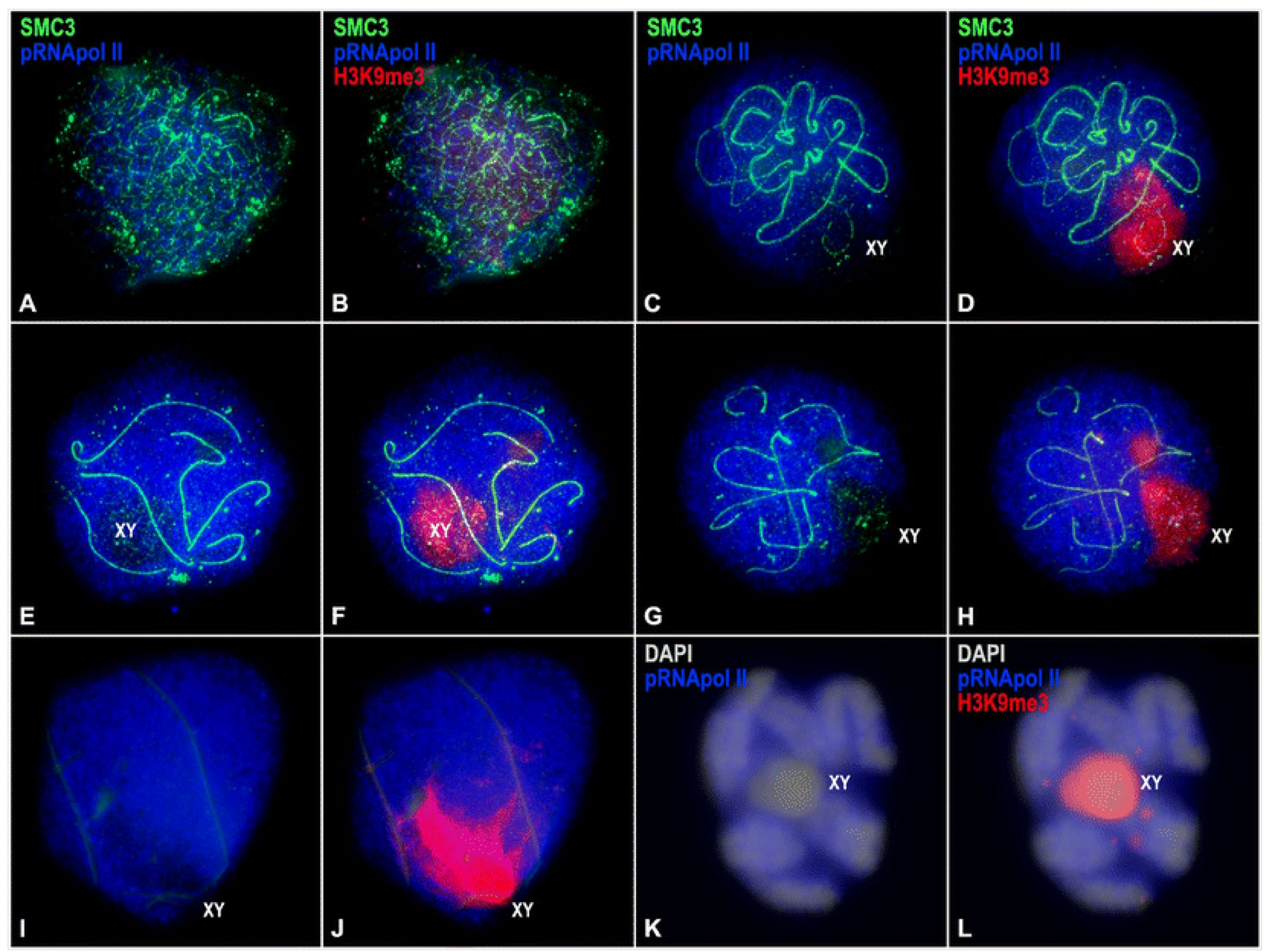

Discussion 
The relationship between synapsis and transcription has been a matter of discussion in the last years. Following the characterisation of MSUC/MSCI mechanisms, which in mammals and worms mainly operate through the epigenetic modifications of the chromatin (Baarends et al., 2005 ; Kelly and Aramayo, 2007 ; Kelly et al., 2002 ; Turner et al., 2005 ), it was proposed that synapsis was necessary for the transcriptional activity of chromosomes (Schimenti, 2005 ). However, when the transcriptional activity pattern was compared to the sequence of synapsis in mammals, it was revealed that transcription is not an immediate consequence of synapsis completion (Page et al., 2012 ) and it was suggested that other factors may have an influence on transcription regulation (Inagaki et al., 2010 ; Page et al., 2012). Our results from the analysis of transcriptional patterns during meiosis in two species of hemipterans reported here add new and interesting elements to this discussion.

\section{The onset of meiosis in hemipterans is a stage of reduced transcription}

Transcription in early stages of meiosis in the two true bugs here analysed seems to be much reduced during leptotene. This fact may be due to several factors. First, it is possible that this reduction is determined by events occurring before the onset of meiosis. The decision to enter meiosis seems to be taken in the previous $\mathrm{G}_{1}$ stage and is accompanied by the expression of specific regulatory proteins like STRA8 (Anderson et al., 2008 ), the loading of the meiosis-specific cohesin REC8 onto chromosomes (Watanabe and Nurse 1999 ) and a longer duration of the S phase (Cha et al., 2000 ; Holm, 1977 ; Jaramillo-Lambert et al., 2007 ), due to a delayed firing of replication origins and/or to a lower efficiency of replication progression (Blitzblau et al., 2012 ). Thus, one can speculate that a stage of transcriptional low activity following the premeiotic $\mathrm{S}$ phase could also influence the regulation of meiosis entry. Second, the leptotene stage is characterised by a program of massive DNA DSBs, performed by SPO11 protein (Keeney et al., 1997 ; Keeney and Neale, 2006 ). These DSBs are usually accompanied by a silencing of the chromatin surrounding them. In mammals, this damage is typically signalled by the phosphorylation of histone variant $\mathrm{H} 2 \mathrm{AX}(\gamma \mathrm{H} 2 \mathrm{AX})$, which rapidly expands to large regions of the nucleus (Mahadevaiah et al., 2001 ). Likewise, the onset of meiosis in grasshoppers is characterised by the presence of high levels of $\gamma \mathrm{H} 2 \mathrm{AX}$, although with a much reduced number of RAD51 foci if compared to that found in mammals (Calvente et al., 2005 ; Viera et al., 2004a; Viera et al., 2009b ; Viera et al., 2010). In both cases, DNA repair 
may be related to the reduction or absence of transcription observed in leptotene. Although we could not find any labelling with anti- $\gamma \mathrm{H} 2 \mathrm{AX}$ antibodies in the species here analysed, it seems clear that in this case, meiosis initiation is accompanied by the presence of DNA DSBs and DNA repair mechanisms, as revealed by the presence of RAD51 associated to chromosomes during leptotene (Fig. 4 ; Viera et al., 2009a). Thus, it is reasonable to postulate that in hemipterans, DNA repair at the beginning of meiosis is accompanied by a low transcription state.

\section{AQ2}

Although a low transcriptional activity at the beginning of meiosis has been reported in mammals (Monesi, 1965 ; Page et al., 2012 ), grasshoppers (Das et al., 1965 ; Henderson, 1963 ) and hemipterans (this work), some invertebrate species deviate from this pattern, indicating that this feature must not be considered universal for animal meiosis. Thus, transcription seems to remain constant and high in C. elegans autosomes from premeiotic stages up to diplotene, both in spermatogenesis and oogenesis (Chu and Shakes, 2013; Kelly et al., 2002; Maine, 2010; Shakes et al., 2009). Likewise, Drosophila males show a rather constant transcriptional activity throughout the first meiotic prophase (Hennig, 1968 ; Hennig and Weyrich, 2013 ; Kremer et al., 1986 ). Nevertheless, the behaviour shown by these two species can be related to other particular features as regards the meiosis initiation. For instance, synapsis precedes induction of DSBs in C. elegans (Dernburg et al., 1998 ). This sequence of events is the reverse to that described in mammals (Mahadevaiah et al., 2001 ), grasshoppers (Viera et al., 2004b ) and the hemipterans here studied. The meiosis of Drosophila males is a paradigm of weird features, including the absence of synapsis and recombination (reviewed in (McKee et al., 2012 ). Therefore, we suggest that the initial state of low or inactive transcription can be considered a common situation in animals and that the particular features of both C. elegans and Drosophila may represent a part of a highly modified pattern of early meiosis events.

\section{Transcription reactivation precedes synapsis completion in true bugs}

Following the initial low transcription stage, transcription in true bugs, as revealed by the presence of RNA polymerase II, reactivates during zygotene and is maintained until the late stages of the first meiotic prophase. These results agree well with the transcription patterns previously described in grasshoppers (Das et al., 1965 ; Henderson, 1963 ), 
indicating that its regulation in these insect groups clearly differs from the mammalian model. The early transcription reactivation during zygotene demonstrates that it is not dependent on synapsis completion, since it involves synapsed and unsynapsed chromosome regions. Moreover, C. fuscispinus, that shows synapsis delay, exhibits a simultaneous or even earlier incorporation of RNApol II to chromosomes if compared to that of G. italicum. These facts indicate that the timing of transcription regulation in hemipterans is quite different from that reported in mammals.

Since the transcription hemipterans can be reactivated in the presence of abundant unsynapsed chromosome domains, the dependence of transcription reactivation on the completion of synapsis cannot be considered a general rule. Moreover, this is also coincident with the presence of abundant DNA repair activity in the nucleus during zygotene, indicating that in these species transcription and DNA repair are not exclusive processes, as suggested for mammals (Inagaki et al., 2010 ; Page et al., 2012 ). How can these differences be explained? The silencing of chromosomes in mammals from leptotene up to early pachytene seems to be associated to different epigenetic modifications. The main factor is the presence of $\gamma \mathrm{H} 2 \mathrm{AX}$ that appears in response to the induction of DNA DSBs (Mahadevaiah et al., 2001 ), but this is preceded by the accumulation of other histone modifications (van der Heijden et al., 2007 ), including H3K9me3 and H3K4me (Page et al., 2012 ). None of these epigenetic marks were detected in bugs during early meiosis: $\gamma \mathrm{H} 2 \mathrm{AX}$ and $\mathrm{H} 3 \mathrm{~K} 4 \mathrm{me}$ antibodies do not yield any labelling, while $\mathrm{H} 3 \mathrm{~K} 9 \mathrm{me} 3$ is only concentrated at the ends of some chromosomes and covers the sex chromosomes from leptotene onwards. Indeed, this $\mathrm{H} 3 \mathrm{~K} 9 \mathrm{me} 3$ labelling pattern likely represents constitutive chromatin modifications, rather than a specific meiotic silencing mark, since it is also present in spermatogonial cells. Therefore, the absence of these repressive chromatin modifications at the beginning of meiosis may lead to an earlier reactivation of transcription. Therefore, it is reasonable to propose that the early meiotic epigenetic patterns, together with the synaptic state (Schimenti, 2005 ; Turner et al., 2005 ) and DNA repair mechanisms (Inagaki et al., 2010 ; Page et al., 2012 ), are important factors regulating the onset of transcription during meiosis.

\section{Sex chromosomes trigger a non-canonical MSCI response}

Sex chromosomes also seem to display an MSCI response different from that previously reported in mammals (Handel, 2004 ; Handel and Hunt, 1992 ; Hoyer-Fender, 2003 ; Solari, 1974 ) and grasshoppers (Cabrero et al., 2007 ; Henderson, 
1963 ; Viera et al., 2004a; Viera et al., 2004b ). X and Y chromosomes remain asynapsed in the two species here analysed and they are clearly excluded from the incorporation of RNA polymerase II at zygotene. However, the accumulation of $\mathrm{H} 3 \mathrm{~K} 9 \mathrm{me} 3$ on the sex chromosomes seems to be a constitutive mark during meiosis and is also present in premeiotic spermatogonial cells. This epigenetic mark is commonly associated to heterochromatin (Cowell et al., 2002 ). Thus, the presence of $\mathrm{H} 3 \mathrm{~K} 9 \mathrm{me} 3$ on the sex chromosomes already in leptotene may not be related to a meiosis-specific function, but to a constitutive chromatin modification of the sex chromosomes. H3K9me3 has been involved in MSCI in mammals (Page et al., 2012 ; van der Heijden et al., 2007 ), although the timing and pattern of localisation are not identical to those of bugs: in mammals, H3K9me3 presents a fluctuating appearance and disappearance. This has been explained as a consequence of $\mathrm{H} 3$ variant replacements during the first meiotic prophase in mouse, a feature that has also been reported in Drosophila (Hennig and Weyrich, 2013 ). Thus, the epigenetic changes associated to MSCI seem to be very variable between species, although all of them would finally achieve the inactivation of the sex chromosomes.

Our results open other interesting questions about the nature of sex chromosome inactivation. We have shown that the relationship between the existence of non-synapsed regions and inactivation is not as straightforward in bugs as it is in other animals. This fact is reinforced by the observation that both synapsed and unsynapsed autosomal regions resume transcription simultaneously. On the other hand, our results also show that MSCI in bugs could be just a manifestation of the constitutive inactivation of the sex chromosomes. Thus, one can confidently speculate that MSCI in animals is not only due to asynapsis. Indeed, in C. elegans oogenesis the X chromosomes are subjected to MSCI despite they achieve complete synapsis (Kelly and Aramayo, 2007 ; Kelly et al., 2002 ; Maine, 2010 ). Similarly, in mammals, the heterologous synapsis of $\mathrm{X}$ and $\mathrm{Y}$ chromosomes at the beginning of pachytene does not preclude the inactivation of sex chromosomes (Page et al., 2012 ). By contrast, the asynaptic sex chromosomes of Drosophila males seem to be transcriptionally active during the first meiotic prophase (Hennig and Weyrich, 2013 ; Kremer et al., 1986 ). In this sense, distinct processes, other than asynapsis, have been suggested to be involved in the sex chromosome inactivation. These include the repression of genes whose expression could be harmful during meiosis (Royo et al., 2010) or the necessity of an inert state of the sex chromosomes for avoiding interactions with the DNA repair machinery (Handel and Hunt, 1992 ; McKee and Handel, 1993 ). In summary, many aspects of the sex chromosome inactivation as well as the 
relationships between synapsis, transcription and epigenetics in meiosis remain to be elucidated. Cumulative knowledge from many other species will help to understand the rationale behind meiotic silencing.

\section{Acknowledgments}

We would like to express our gratitude to Consejería de Medio Ambiente y Ordenación del Territorio (Comunidad de Madrid; España) for emitting the authorization for animal recollections in natural populations; to Dr. José Luis Barbero and Dr. Kim McKim for their generous supply of antibodies; and Dr. Carlos Garcia de la Vega for the critical review of the manuscript. This work was supported by grants BFU2009-10987 from Ministerio de Ciencia e Innovación and grants CGL2014-53106-P and BFU2014-53681-P from Ministerio de Economía y Competitividad (Spain).

Compliance with ethical standards

All experiments performed in this study involving animals were in accordance with the ethical standards of the Universidad Autónoma de Madrid and Comunidad de Madrid (Spain). Collection of animals was authorised by permission Ref. 10/516390.9/11 JML/ecc. The use of insect species is not subjected to approval of the Ethical Committees. Nevertheless, no experimental procedures were applied to any animal.

Conflict of interest The authors declare that they have no competing interests.

\section{Electronic supplementary material}

Below is the link to the electronic supplementary material.

\section{Supplementary Figure 1}

Immunolabelling of H3K9me3 (red) in squashed prometaphase spermatogonial cells of G. italicum counterstained with DAPI (blue). The position of the sex chromosomes is indicated (XY). The image presents 10 focal planes across the equatorial region of two prometaphase spermatogonial cells. H3K9me3 can be detected at discrete domains at the chromosome ends (arrows) and as large accumulations covering the chromatin of the sex chromosomes (XY). (TIF $2500 \mathrm{~kb}$ ) 


\section{Supplementary Video 1}

Immunolabelling of pRNApol II (green) in squashed spermatocytes of G. italicum counterstained with DAPI (pseudo coloured in red). 3D-reconstruction of 35 focal planes across diffuse stage and metaphase I spermatocytes. The positions of the sex chromosomes $(\mathrm{XY})$ and the nucleolus $(\mathrm{Nu})$ are indicated. Note the high levels of pRNApol II in late prophase I spermatocytes, but its absence at metaphase I. For the correct visualisation of the video, please click the loop/boucle option in your video player before running the video. (MOV $1252 \mathrm{~kb}$ )

\section{Supplementary Video 2}

Immunolabelling of H3K9me3 (red) in squashed diplotene nuclei of G. italicum counterstained with DAPI (blue). 3Dreconstruction of 20 focal planes across the entire volume of three spermatocytes. Note that the stage of these spermatocytes is comparable to that shown in Fig. $2 \mathrm{k}$ and 1 . As the nuclear volume is preserved, this video facilitates the visualisation of the individualising autosomal bivalents. To ensure the proper identification of H3K9me3 at both chromosome ends and over the sex chromosomes (XY) the conditions for imaging capture were optimised for these specific spermatocytes. For the correct visualisation of the video, please click the loop/boucle option in your video player before running the video. (MOV $1029 \mathrm{~kb})$ 


\section{Supplementary Video 3}

Double immunolabelling of SMC3 (green) and H3K9me3 (red) in squashed prophase I spermatocytes and spermatids of $G$. italicum. Leptotene (Le), Zygotene (Zy), Pachytene (Pa) and Diffuse stage (Diffuse) spermatocytes are shown, along with a spermatid (Sp). 3D-reconstruction of 20 focal planes across prophase I spermatocytes. Please note that part of this field is shown in Fig. 3 . Independently of the prophase I stage, recognised by the state of SMC3 axes (see text for further information), H3K9me3 is accumulated as discrete foci in the chromosome ends and as large accumulations in the chromatin of the sex chromosomes. For the correct visualisation of the video, please click the loop/boucle option in your video player before running the video. (MOV $1397 \mathrm{~kb}$ )

\section{Supplementary Video 4}

Immunolabelling of $\mathrm{H} 3 \mathrm{~K} 9 \mathrm{me} 3$ (red) in squashed prometaphase spermatogonial cells of G. italicum counterstained with DAPI (blue). Animation of 20 focal planes across prometaphase spermatogonial cells. Please note that part of this field is shown in Supplementary Figure 1. In all cells H3K9me3 can be found as discrete accumulations at the chromosome ends and as large masses over the sex chromosomes. For the correct visualisation of the video, please click the loop/boucle option in your video player before running the video. (MOV $999 \mathrm{~kb}$ )

\section{References}

Anderson EL, Baltus AE, Roepers-Gajadien HL, Hassold TJ, de Rooij DG, van Pelt AMM, Page DC (2008) Stra8 and its inducer, retinoic acid, regulate meiotic initiation in both spermatogenesis and oogenesis in mice. Proc Natl Acad Sci U S A 105:14976-14980

Baarends WM, Wassenaar E, van der Laan R, Hoogerbrugge J, Sleddens-Linkels E, Hoeijmakers JH, de Boer P, Grootegoed JA (2005) Silencing of unpaired chromatin and histone H2A ubiquitination in mammalian meiosis. Mol 
Blitzblau HG, Chan CS, Hochwagen A, Bell SP (2012) Separation of DNA replication from the assembly of breakcompetent meiotic chromosomes. PLoS Genet 8:e1002643

Cabrero J, Teruel M, Carmona FD, Jimenez R, Camacho JP (2007) Histone H3 lysine 9 acetylation pattern suggests that $\mathrm{X}$ and $\mathrm{B}$ chromosomes are silenced during entire male meiosis in a grasshopper. Cytogenet Genome Res 119:135-142

Calvente A, Viera A, Page J, Parra MT, Gomez R, Suja JA, Rufas JS, Santos JL (2005) DNA double-strand breaks and homology search: inferences from a species with incomplete pairing and synapsis. J Cell Sci 118:2957-2963

Costa Y, Speed RM, Gautier P, Semple CA, Maratou K, Turner JM, Cooke HJ (2006) Mouse MAELSTROM: the link between meiotic silencing of unsynapsed chromatin and microRNA pathway? Hum Mol Genet 15:2324-2334

Cowell IG, Aucott R, Mahadevaiah SK, Burgoyne PS, Huskisson N, Bongiorni S, Prantera G, Fanti L, Pimpinelli S, Wu R, Gilbert DM, Shi W, Fundele R, Morrison H, Jeppesen P, Singh PB (2002) Heterochromatin, HP1 and methylation at lysine 9 of histone $\mathrm{H} 3$ in animals. Chromosoma 111:22-36

Cha RS, Weiner BM, Keeney S, Dekker J, Kleckner N (2000) Progression of meiotic DNA replication is modulated by interchromosomal interaction proteins, negatively by Spo11p and positively by Rec8p. Genes Dev 14:493-503

Chu DS, Shakes DC (2013) Spermatogenesis. Adv Exp Med Biol 757:171-203

Das NK, Siegel EP, Alfert M (1965) Synthetic activities during spermatogenesis in the locust. J Cell Biol 25:387395 
Dernburg AF, McDonald K, Moulder G, Barstead R, Dresser M, Villeneuve AM (1998) Meiotic recombination in C. elegans initiates by a conserved mechanism and is dispensable for homologous chromosome synapsis. Cell 94:387-398

Eijpe M, Heyting C, Gross B, Jessberger R (2000) Association of mammalian SMC1 and SMC3 proteins with meiotic chromosomes and synaptonemal complexes. J Cell Sci 113(Pt 4):673-682

Fernandez-Capetillo O, Lee A, Nussenzweig M, Nussenzweig A (2004) H2AX: the histone guardian of the genome. DNA Repair (Amst) 3:959-967

Handel MA (2004) The XY body: a specialized meiotic chromatin domain. Exp Cell Res 296:57-63

Handel MA, Hunt PA (1992) Sex-chromosome pairing and activity during mammalian meiosis. Bioessays 14:817-822

Henderson SA (1963) Differential ribonucleic acid synthesis of X and autosomes during meiosis. Nature 200:1235

Hennig W (1968) Ribonucleic acid synthesis of the Y-chromosome of Drosophila hydei. J Mol Biol 38:227-239

Hennig W, Weyrich A (2013) Histone modifications in the male germ line of Drosophilaa. BMC Dev Biol 13:7

Hirano T (2002) The ABCs of SMC proteins: two-armed ATPases for chromosome condensation, cohesion, and repair. Genes Dev 16:399-414

Holm P (1977) The premeiotic DNA replication of euchromatin and heterochromatin in Lilium longiflorum (Thunb.). Carlsberg Res Commun 42:249-281

Hoyer-Fender S (2003) Molecular aspects of XY body formation. Cytogenet Genome Res 103:245-255 
Inagaki A, Schoenmakers S, Baarends WM (2010) DNA double strand break repair, chromosome synapsis and transcriptional silencing in meiosis. Epigenetics 5:255-266

James RD, Schmiesing JA, Peters AH, Yokomori K, Disteche CM (2002) Differential association of SMC1alpha and SMC3 proteins with meiotic chromosomes in wild-type and SPO11-deficient male mice. Chromosome Res 10:549-560

Jaramillo-Lambert A, Ellefson M, Villeneuve AM, Engebrecht J (2007) Differential timing of S phases, X chromosome replication, and meiotic prophase in the C. elegans germ line. Dev Biol 308:206-221

Keeney S, Giroux CN, Kleckner N (1997) Meiosis-specific DNA double-strand breaks are catalyzed by Spo11, a member of a widely conserved protein family. Cell 88:375-384

Keeney S, Neale MJ (2006) Initiation of meiotic recombination by formation of DNA double-strand breaks: mechanism and regulation. Biochem Soc Trans 34:523-525

Kelly WG, Aramayo R (2007) Meiotic silencing and the epigenetics of sex. Chromosome Res 15:633-651

Kelly WG, Schaner CE, Dernburg AF, Lee MH, Kim SK, Villeneuve AM, Reinke V (2002) X-chromosome silencing in the germline of C. elegans. Development 129:479-492

Kierszenbaum AL, Tres LL (1974) Nucleolar and perichromosomal RNA synthesis during meiotic prophase in the mouse testis. J Cell Biol 60:39-53

Kota SK, Feil R (2010) Epigenetic transitions in germ cell development and meiosis. Dev Cell 19:675-686

Kremer H, Hennig W, Dijkhof R (1986) Chromatin organization in the male germ line of Drosophila hydei. 
Mahadevaiah SK, Turner JM, Baudat F, Rogakou EP, de Boer P, Blanco-Rodriguez J, Jasin M, Keeney S, Bonner WM, Burgoyne PS (2001) Recombinational DNA double-strand breaks in mice precede synapsis. Nat Genet 27:271-276

Maine EM (2010) Meiotic silencing in Caenorhabditis elegans. Int Rev Cell Mol Biol 282:91-134

Manterola M, Page J, Vasco C, Berrios S, Parra MT, Viera A, Rufas JS, Zuccotti M, Garagna S, Fernandez-Donoso R (2009) A high incidence of meiotic silencing of unsynapsed chromatin is not associated with substantial pachytene loss in heterozygous male mice carrying multiple simple robertsonian translocations. PLoS Genet 5:e1000625

Matsui Y, Hayashi K (2007) Epigenetic regulation for the induction of meiosis. Cell Mol Life Sci 64:257-262

McKee B, Handel M (1993) Sex chromosomes, recombination, and chromatin conformation. Chromosoma 102:71-80

McKee BD, Yan R, Tsai JH (2012) Meiosis in male Drosophila. Spermatogenesis 2:167-184

Monesi V (1964) Ribonucleic acid synthesis during mitosis and meiosis in the mouse testis. J Cell Biol 22:521-532

Monesi V (1965) Differential rate of ribonucleic acid synthesis in the autosomes and sex chromosomes during male meiosis in the mouse. Chromosoma 17:11-21

Page J, Berrios S, Rufas JS, Parra MT, Suja JA, Heyting C, Fernandez-Donoso R (2003) The pairing of X and Y chromosomes during meiotic prophase in the marsupial species Thylamys elegans is maintained by a dense plate developed from their axial elements. J Cell Sci 116:551-560 
Page J, de la Fuente R, Manterola M, Parra MT, Viera A, Berrios S, Fernandez-Donoso R, Rufas JS (2012) Inactivation or non-reactivation: what accounts better for the silence of sex chromosomes during mammalian male meiosis? Chromosoma 121:307-326

Page J, Suja JA, Santos JL, Rufas JS (1998) Squash procedure for protein immunolocalization in meiotic cells. Chromosome Res 6:639-642

Parra MT, Page J, Yen TJ, He D, Valdeolmillos A, Rufas JS, Suja JA (2002) Expression and behaviour of CENP-E at kinetochores during mouse spermatogenesis. Chromosoma 111:53-61

Pelttari J, Hoja MR, Yuan L, Liu JG, Brundell E, Moens P, Santucci-Darmanin S, Jessberger R, Barbero JL, Heyting C, Hoog C (2001) A meiotic chromosomal core consisting of cohesin complex proteins recruits DNA recombination proteins and promotes synapsis in the absence of an axial element in mammalian meiotic cells. Mol Cell Biol 21:5667-5677

Peters AH, Plug AW, van Vugt MJ, de Boer P (1997) A drying-down technique for the spreading of mammalian meiocytes from the male and female germline. Chromosome Res 5:66-68

Rebagliati PJ, Mola LM, Papeschi AG, Grazia J (2005) Cytogenetic studies in Pentatomidae (Heteroptera): a review. Cytogetische Untersuchungen an Pentatomidae (Heteroptera). Ein review. J Zool Syst Evol Res 43:199-213

Royo H, Polikiewicz G, Mahadevaiah SK, Prosser H, Mitchell M, Bradley A, de Rooij DG, Burgoyne PS, Turner JM (2010) Evidence that meiotic sex chromosome inactivation is essential for male fertility. Curr Biol 20:2117-2123

Schimenti J (2005) Synapsis or silence. Nat Genet 37:11-13

Schoenmakers S, Wassenaar E, Hoogerbrugge JW, Laven JSE, Grootegoed JA, Baarends WM (2009) Female meiotic 
sex chromosome inactivation in chicken. PLoS Genet 5:e1000466

Shakes DC, Wu JC, Sadler PL, Laprade K, Moore LL, Noritake A, Chu DS (2009) Spermatogenesis-specific features of the meiotic program in Caenorhabditis elegans. PLoS Genet 5:e1000611

Shiu PK, Raju NB, Zickler D, Metzenberg RL (2001) Meiotic silencing by unpaired DNA. Cell 107:905-916

Solari AJ (1974) The behavior of the XY pair in mammals. Int Rev Cytol 38:273-317

Turner JM (2007) Meiotic sex chromosome inactivation. Development 134:1823-1831

Turner JM, Mahadevaiah SK, Fernandez-Capetillo O, Nussenzweig A, Xu X, Deng CX, Burgoyne PS (2005)

Silencing of unsynapsed meiotic chromosomes in the mouse. Nat Genet 37:41-47

van der Heijden GW, Derijck AA, Posfai E, Giele M, Pelczar P, Ramos L, Wansink DG, van der Vlag J, Peters AH, de Boer P (2007) Chromosome-wide nucleosome replacement and H3.3 incorporation during mammalian meiotic sex chromosome inactivation. Nat Genet 39:251-258

Viera, A., A. Calvente, J. Page, M.T. Parra, R. Gomez, J.A. Suja, J.S. Rufas, and J.L. Santos (2004a) X and B chromosomes display similar meiotic characteristics in male grasshoppers. Cytogenet Genome Res. 106:302-308

Viera, A., J. Page, and J.S. Rufas (2009c) Inverted meiosis: the true bugs as a model to study. Genome Dyn. 5:137-156

Viera A, Parra MT, Page J, Santos JL, Rufas JS, Suja JA (2003) Dynamic relocation of telomere complexes in mouse meiotic chromosomes. Chromosome Res 11:797-807 
Viera, A., J.L. Santos, J. Page, M.T. Parra, A. Calvente, M. Cifuentes, R. Gomez, R. Lira, J.A. Suja, and J.S. Rufas (2004b) DNA double-strand breaks, recombination and synapsis: the timing of meiosis differs in grasshoppers and flies. EMBO Rep. 5:385-591

Viera A, Santos JL, Parra MT, Calvente A, Gomez R, de la Fuente R, Suja JA, Page J, de la Vega CG, Rufas JS (2010) Incomplete synapsis and chiasma localization: the chicken or the egg? Cytogenet Genome Res 128:139-151

Viera, A., J.L. Santos, M.T. Parra, A. Calvente, R. Gomez, R. de la Fuente, J.A. Suja, J. Page, and J.S. Rufas (2009a) Cohesin axis maturation and presence of RAD51 during first meiotic prophase in a true bug. Chromosoma 118:575-589

Viera, A., J.L. Santos, and J.S. Rufas (2009b) Relationship between incomplete synapsis and chiasma localization. Chromosoma 118: 377-389

Watanabe Y, Nurse P (1999) Cohesin Rec8 is required for reductional chromosome segregation at meiosis. Nature 400:461-464 